\title{
Retraction Note to: Advanced oxidation protein products induce chondrocyte death through a redox-dependent, poly(ADP-ribose) polymerase-1-mediated pathway
}

\author{
Wenbin $\mathrm{Ye}^{1} \cdot$ Zhaoming Zhong $^{2} \cdot$ Siyuan $\mathrm{Zhu}^{2} \cdot$ Shuai Zheng ${ }^{2} \cdot$ Jun Xiao $^{3} \cdot$ \\ Shaolian Song ${ }^{4}$ Hui $\mathrm{Yu}^{2} \cdot$ Qian $\mathrm{Wu}^{2} \cdot{\mathrm{Zhen} \mathrm{Lin}^{2} \cdot \text { Jianting Chen }}^{2}$
}

Published online: 18 April 2017

(C) Springer Science+Business Media New York 2017

\section{Retraction Note to: Apoptosis (2017) 22:86-97 DOI 10.1007/s10495-016-1314-6}

The authors have requested to retract their paper which had already been online, entitled: 'Advanced oxidation protein products induce chondrocyte death through a redoxdependent, poly (ADP-ribose) polymerase-1-mediated pathway'. DOI 10.1007/s10495-016-1314-6. Their paper reported the advanced oxidation protein products (AOPPs) stimulation cause cell death of human chondrocyte of RA patients with PARP-1 over-expression and characterized the apoptosis mechanisms underlying the redox condition. Many of the figure panels in the paper summarize data from multiple experiments. The authors have now detected a number of errors in these panels. The authors believe that the most responsible course of action is to retract our paper. The authors sincerely apologize for their carelessness and poor arrangement of results and apologize to the community.

The online version of the original article can be found under doi:10.1007/s10495-016-1314-6.

Jianting Chen

jianting_chen@sina.com

1 Department of Orthopaedics, The 175th Hospital of PLA, Affiliated Dongnan Hospital of Xiamen University, Zhangzhou, Fujian, People's Republic of China

2 Department of Orthopaedic Spinal Surgery, Nanfang Hospital, Southern Medical University, 1838 North Guangzhou Avenue, Guangzhou 510515, People's Republic of China

3 Department of Orthopedic Joint Surgery, Nanfang Hospital, Southern Medical University, Guangzhou, People's Republic of China

4 Department of Pharmacy, Nanfang Hospital, Southern Medical University, Guangzhou, People's Republic of China 